\title{
Development of a Competitive and Collaborative Platform for Block Diagram and Resistive Circuit Reduction in a Basic Electrical Engineering Course
}

\author{
Tolulope Odetola, Obasegun Ayodele, Caleb Onigbinde,, Lawrence O. Kehinde* \\ Department of Electronic and Electrical Engineering \\ Obafemi Awolowo University \\ Ile-Ife, NIGERIA \\ *lkehinde@oauife.edu.ng
}

\begin{abstract}
Competition and collaboration are two universal ingredients in all human cultures. Competition has been observed over time as a phenomenon that has helped people achieve the very best of their potentials in their various fields. Competition has also helped individuals achieve feats they would not have ordinarily achieved as they are pushed beyond their limits. The same can also be said of cooperation and collaboration, which can be used to achieve specific goals or objectives. Hence, both competition and collaboration can be vital in creating a healthy learning process among students.

This paper discusses a competitive and collaborative platform on the learning process for two selected online courses in Electrical Engineering. Using a platform developed in LabVIEW programming language, a community of students were encouraged to compete online as they try to reduce block diagrams of subsystems in Control Engineering as well as reduce given Series-Parallel Resistor Circuits in the shortest time and amount of steps possible. At the end of each trial, students can view the finish time of other students and can share, online, their individual solutions with the community as well as discuss ideas such as reduction techniques they find most expedient via a public online notepad. This creates a means of learning from the community. The platform helps to passively induce a competitive and collaborative perspective in the learning pathway of students, thereby improving the interest and experience of students in the selected subject areas. The platform uses an online server to maintain communications from all the students in their various locations. Students can view the timed performance of their counterparts on a leadership board thereby engendering induced competition in the learning community. The instructor or administrator can also monitor activities in the community from a server portal.
\end{abstract}

Keywords - competition, collaboration; Electrical Engineering; Block Diagrams; reduction; LabView; reduction

\section{INTRODUCTION}

According to the constructivist learning theory people generate knowledge and meaning when they share their ideas and experiences from the interaction between them [1]. This paper introduces a platform that offers this interaction to students and provides a new approach that uses minimal steps for the reduction, of block diagrams of subsystems as well as series-parallel resistor circuits in a basic Electrical and Electronic Engineering course. Basic block diagram and circuit reduction rules are used and it is expected that students would have covered these in class before attempting the exercises.

\section{OVERVIEW OF BLOCK DIAGRAM AND SERIES-PARALLEL RESISTOR CIRCUITS REDUCTION}

\section{A. Block Diagram Reduction}

Block diagrams represent components of the transfer function of control subsystems. A control system may consist of multiple subsystems interconnected by summing junctions and pickoff points [2-5]. Blocks may be interconnected either in cascade form, parallel form or in feedback form. . They can be used together with transfer functions to describe cause and affect relationships in a system [6]. Block diagrams can be simplified by relocating individual blocks using well known rules. Table 1 shows well known examples of relocation and simplification of some block diagrams. There are many more in literature and students' textbooks provide these.

TABLE 1: Manipulation and Simplification of Basic Block Diagrams

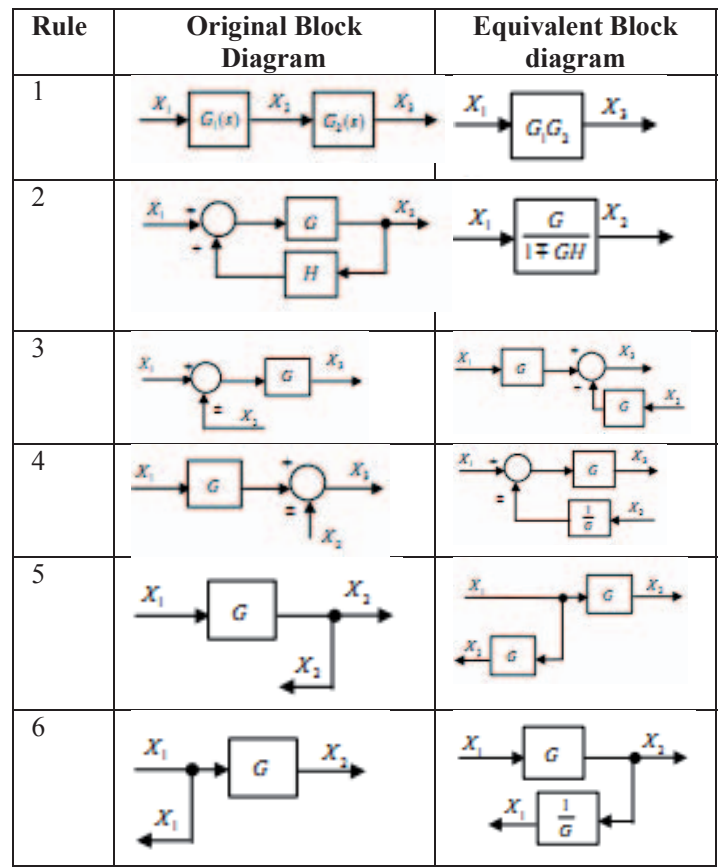


B. Overview of Resistor Circuit Component Simplification

The basic components in electric circuits are resistors, inductors and capacitors. These components can be connected either in series form or in parallel form to yield the resultant resistance or impedance in a circuit. Table 2 shows a few simple forms of resistor connections and their equivalences.

\section{DESCRIPTION OF EXPERIMENT PLATFORM}

The platform seeks to passively trigger the competitive and collaborative instincts in students, enabling them apply these instincts in their coursework. The platform is an online architecture which is hosted on a google server. The platform is developed using LabVIEW programming language. It also provides an offline practice session for the students to improve their skill on the platform.

\section{A. Platform Features}

The platform has the following features:

- A sign up page

- A sign in page

- A registration form

- A dashboard

- Block diagram category

- Circuit theory category

- Leaderboard

- Collaboration pad

\section{B. Platform Methodology on Block Diagram.}

At program initiation, a splash screen shown in Fig. 1 appears during which proper loading of the program components occurs before the user starts to run any experiment. The splash screen eventually gives way to the sign-in/ sign-up page shown in Fig. 2.

TABLE 2 Manipulation of a few basic resistors

\begin{tabular}{|c|c|}
\hline Original Circuit Diagram & $\begin{array}{c}\text { Equivalent Circuit } \\
\text { Diagram }\end{array}$ \\
\hline & \\
\hline
\end{tabular}

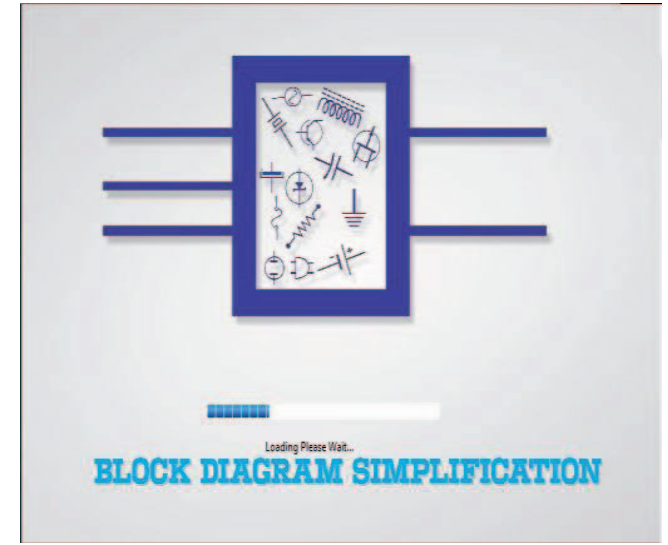

Fig. 1. Splash Screen at Program initiation

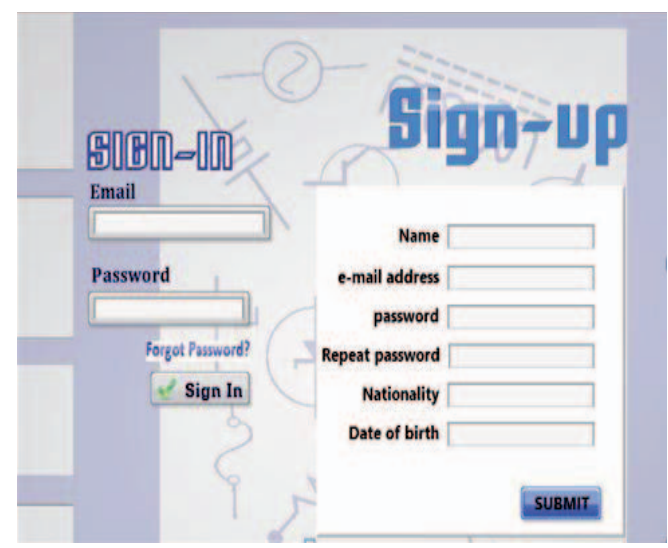

Fig. 2. Detailed Sign-in/ Sign-up page

If the user is in for the first session on the software, the user is required to click on the sign-up button placed beside the sign-in section to get the sign-up form shown. After submitting the form, he or she gets an account which can be accessed at subsequent visits using the details submitted in the sign-up form.

After the proper filling of the appropriate sections on the sign-in/sign-out page, the user is then shown the home page/ dashboard of Fig. 3 on which the main options are provided. At this stage, we only have the block diagram and the circuit theory options. The user gets to choose one of the options and this leads to the level page where he is allowed to choose from the various complexity levels that are provided.

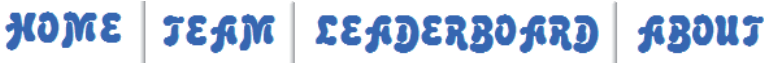

\section{DASHBDARD}

BLOCK DIAGRAM

CIRCUIT DIAGRAM

Fig. 3 Dashboard 
Level 1 is the most basic. The choice of Block diagram option leads to the page shown in Fig. 4. For the block diagram option, different control block diagrams have been programmed into the different levels. If for example, level 1 is selected, the page changes to Fig. 5. The student can then click on any of the blocks from which he will be given several simplification rule hints to choose from. Good selection depends on student's understanding of the basic block diagram reduction rules which must have been taught in class. The platform utilizes selected basic block reduction rules. Clicking on $\mathrm{G}_{1}$ for example gives the options shown in Fig. 6.

Active rules provided in this case are Rule 2, Rule 3 and Rule 5. If the student next clicks on Rule 2, the following page of Fig. 7 appears. On clicking ok, the page shown in Fig. 8 appears after which the shaded circuit box on the right should be clicked if the rule chosen is accepted, if not student can cancel and chose another rule. If the situation in Fig. 8 is accepted, the scenario of Fig. 9 appears. Clicking on either of the two blocks of Fig. 9, a student can continue in the same way until he arrives at the finished stage of Fig. 10.

The number of steps taken to get to the finished stage is indicated on the Leaderboard which accessible to all participants. In the particular case described above, the sequence taken, $\left(\mathbf{G}_{1}\right)$ Rule2 $\rightarrow\left(\mathbf{G}_{\mathbf{2}}\right)$ Rule1 will give a total no of steps of 2. If a step sequence of $\left(\mathbf{G}_{1}\right)$ Rule $\mathbf{5} \rightarrow\left(\mathbf{H}_{.} \mathbf{G}_{1}\right)$ Rule2 $\rightarrow\left(\mathbf{G}_{2}\right)$ Rule1 is taken, simplification will take place in 3 steps.

\section{HOME TEATM LEADERBOARD ABOUT}

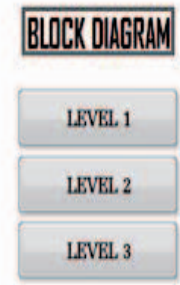

Fig. 4 Block diagram option

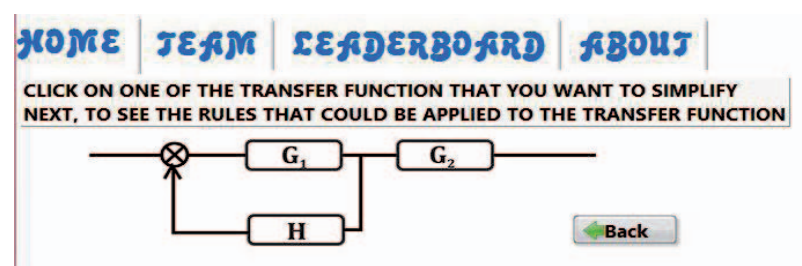

Fig. 5: Level 1 block diagram

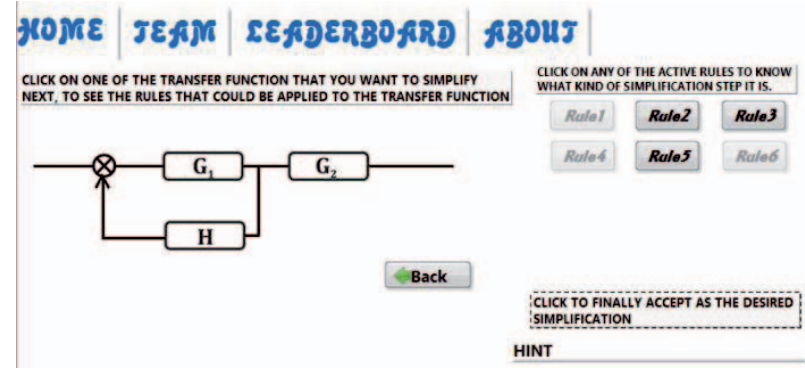

Fig. 6: Options obtained by clicking on $\mathrm{G}_{1}$

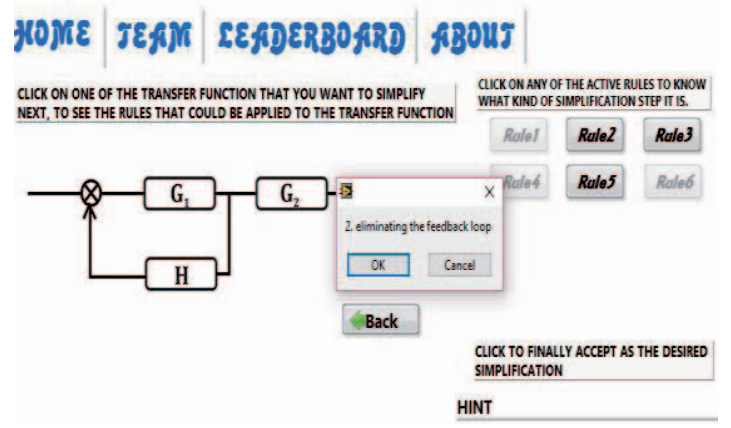

Fig. 7: Display of selected rule2

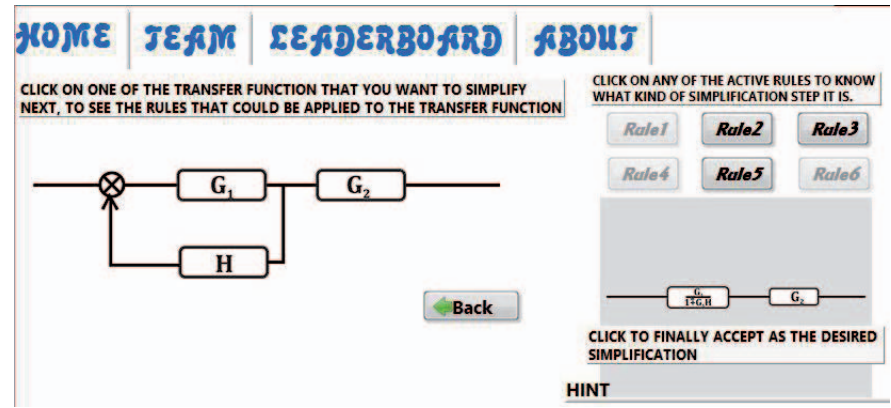

Fig. 8: Display of simplified block diagram

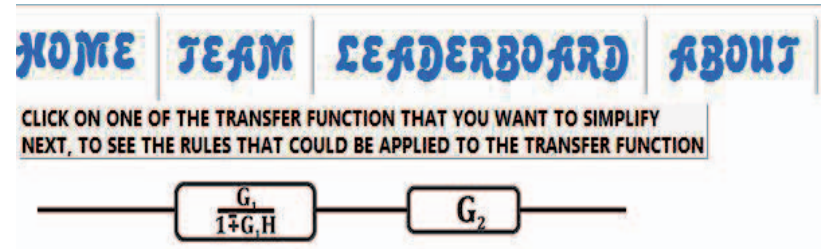

Back

Fig. 9: Accepted simplified block diagram

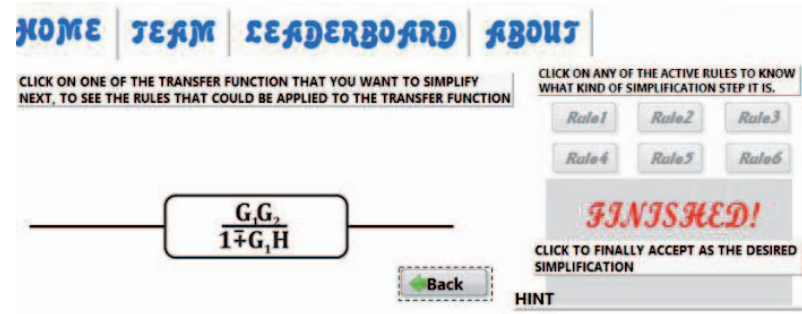

Fig. 10: Finished simplification. 
From the above methodology, there are numerous ways by which different users can select and manipulate each block. Hence, to avoid unwieldiness, it was necessary to limit the user to certain no of rules. For the question above, the user is expected to simplify the block before a maximum specified number of steps (In this case 10 steps). If the block diagram simplification is not successfully completed before this maximum number of steps, the user is prompted to restart the session. The user that simplifies the block diagram in the lowest number of steps will have the highest spot on the leaderboard for that category. The leaderboard provides some form of competition that will motivate the user to look for better ways to simplify the block diagram in lowest number of steps.

The platform also gives the user the privilege to team up and collaborate with like minds to solve problems together via the internet. This acts like a collaborative avenue for the user to help build up his or her problem solving skills and improve his or her knowledge. The performances of the students are graded in order of the amount of steps used to achieve the required simplified transfer function (canonical form).

\section{Platform Methodology For Series-Parallel Resistor Circuit Reduction}

This platform model is similar to the block diagram one, with its rules given beforehand. Only three rules are used in this case as shown in Table 2: Due to page limitations, this example will not be fully described here. Fig. 11 to Fig. 14 show some selected steps in the process of reduction of the resistor circuit of Fig. 11.

\section{THE LEADER BOARD INTERFACE AND COLLABORATION}

The Leader Board feature of the platform is provided to give the user a means of measuring self-performance against the performance of others. The Leader Board will help trigger a competitive perspective to the learning pathway of the students, thereby improving their skills in both block diagram and circuit diagram.

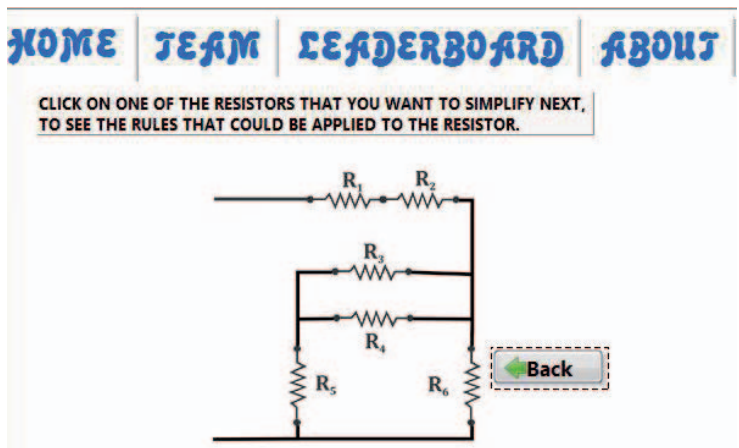

Fig. 11: Resistor circuit to be reduced

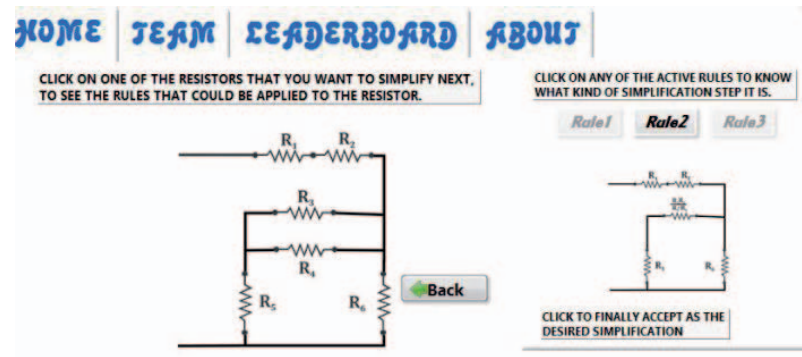

Fig. 12: Intermediate stage

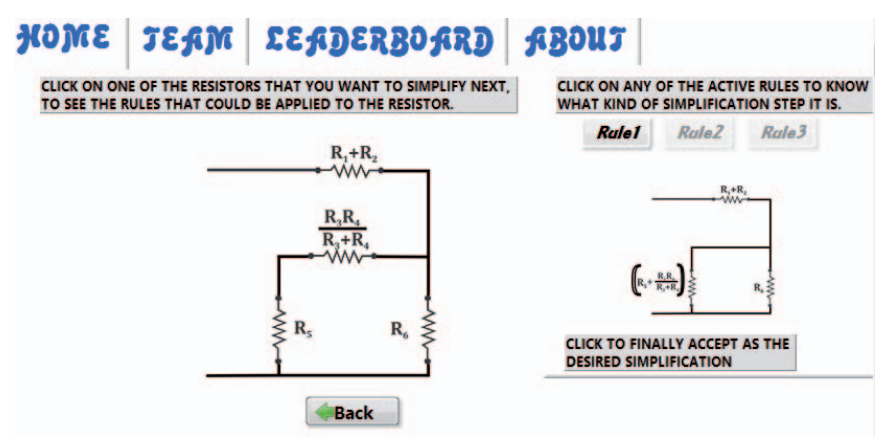

Fig. 13: Another intermediate stage

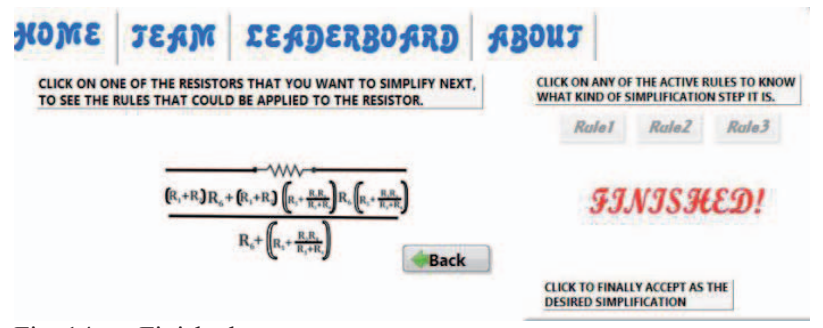

Fig. 14: Finished stage

The Leader Board allows the participant to share and compare their performance in terms of both numbers of step and simplification approach with all other participants using the platform online. The boxed portion in Fig. 15 indicates the view solution button. Individual students can share solutions with other students hereby creating a collaborative means by which knowledge can be shared. The sharing is done by clicking on the share solution button shown in Fig. 16. The top boxed portion in Fig. 16 shows a discussion notepad which provides a means by which students can communicate.

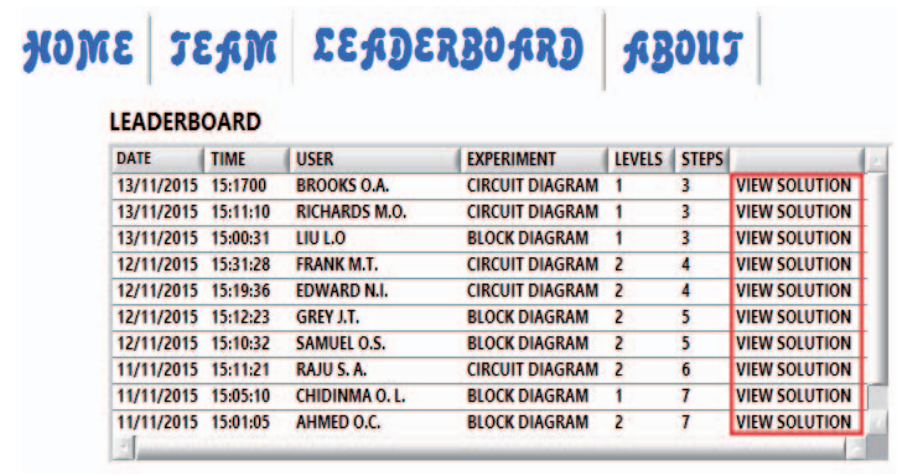

Fig. 15: Leader Board 


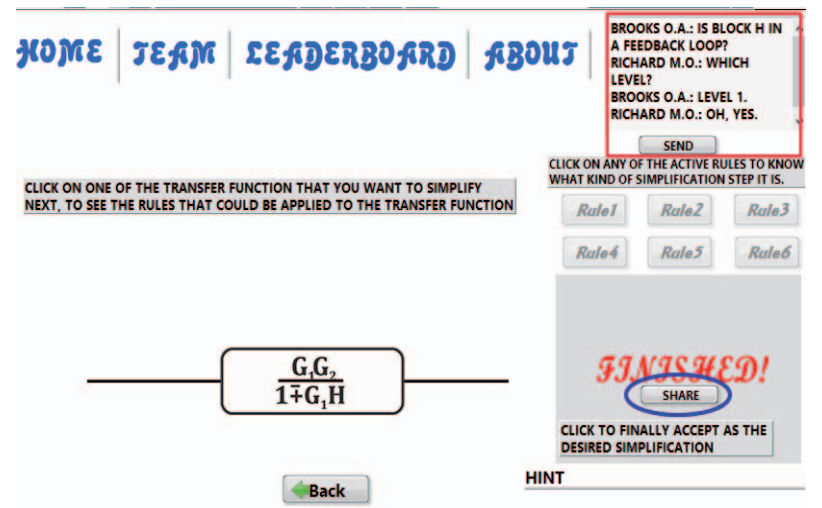

Fig. 16: Public online notepad and share button

\section{RESULT OF STUDENTS'RESPONSES}

To test the effectiveness of the platform, a test experiment was organized. A set of 50 students were provided with the platform. They all started at the same time and at the end a feedback form was filled by each individual student. From Nielsen Heuristics assessment [7], some questions were generated to ensure that the platform was designed to suit requirements for virtual experiments. Fig. 17(a-f) show results of the feedback form from students who used the platform.

From the general overview and statistical analysis of questions relating to Nielsen Heuristic assessment, it shows that the platform is relatively suited to aid learning throughout the process.

\section{CONCLUSIONS}

Figs 18(a-b) give an indication of how much competition and collaboration has been generated by using the platform. Hence, learning can be enhanced by inducing competition and collaboration between participants. In conclusion the leader board provides a platform to create competition or collaboration and hence motivation for learning.

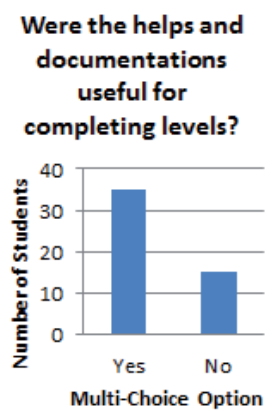

Fig. 17a

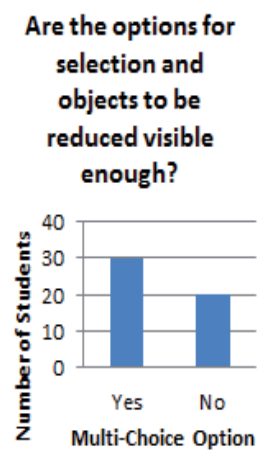

Fig. 17b

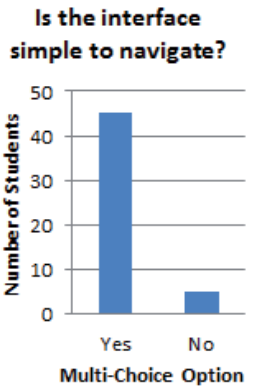

Fig. $17 \mathrm{c}$

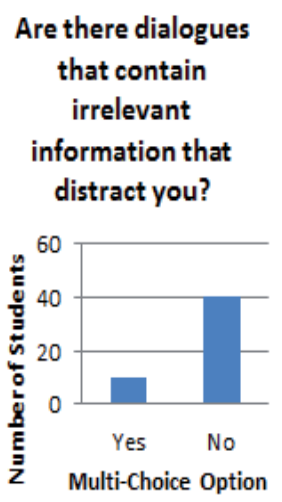

Fig. 17d

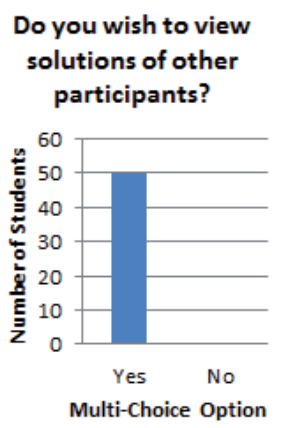

Fig. 17e

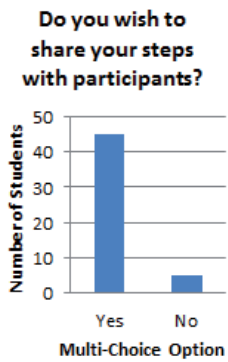

Fig. $17 \mathrm{f}$

Fig. 17: Nielsen Heuristics Assessment Results

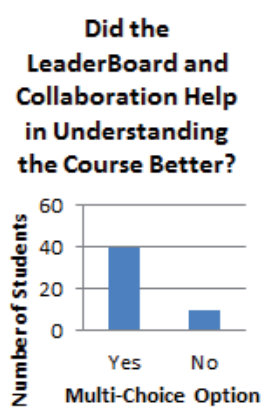

Fig. 18a

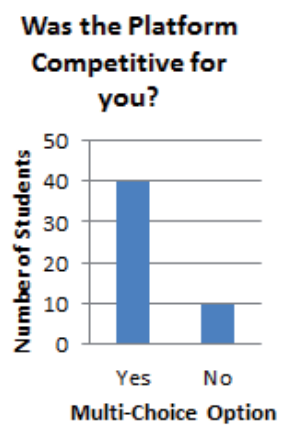

Fig. $18 b$

Fig, 18: Effect of Collaboration and Competition on Learning

\section{REFERENCES}

[1] L. Torre, R. Heradio, C. A. Jara, J. Sanchez, S. Dormido, F. Torres, and F. A. Candelas: Providing Collaborative Support to Virtual and Remote Laboratories (2013).

[2]. R. C. Dorf, Modern Control Systems, 5th Edition, Addison-Wesley Publishing, (1989).

[3]. G. F. Franklin, J. D. Powell and A. Emami-Naeini, Feedback Control Dynamic Systems, $3^{\text {rd }}$ Edition, Addison-Wesley Publishing, (1994).

[4]. K. Ogata, Modern Control Engineering, 3rd Edition, Prentice Hall, Upper Saddle River, New Jersey,(1997).

[5] B. C. Kuo, Automatic Control Systems, 7th Edition, Prentice-Hall, Inc., Upper Saddle A. Bazoune, ME 413: System Dynamics and Control, Time-Domain Analysis and Design of Control Systems: Block Diagram Reduction pp 6 (2007).

C. Mei: "On Teaching the simplifications of Block Diagrams", Int. J. Engng Ed. Vol. 18, No. 6, pp. 697-703, 2002

[7] J. Nielsen, Usability Engineering, Cambridge, MA: Academic Press Professional (1993). 